\title{
Self-duality in quantum impurity problems
}

\author{
P. Fendley ${ }^{1}$ and H. Saleur ${ }^{2}$ \\ 1 Physics Department, University of Virginia, Charlottesville VA 22901 \\ 2 Department of Physics, University of Southern California, Los Angeles CA 90089-0484
}

(April 1998)

\begin{abstract}
We establish the existence of an exact non-perturbative self-duality in a variety of quantum impurity problems, including the Luttinger liquid or quantum wire with impurity. The former is realized in the fractional quantum Hall effect, where the duality interchanges electrons with Laughlin quasiparticles. We discuss the mathematical structure underlying this property, which bears an intriguing resemblance with the work of Seiberg and Witten on supersymmetric non-abelian gauge theory.
\end{abstract}

Duality is a rather ancient concept. It was probably observed first in electromagnetism, where the theory is invariant under exchange of electricity with magnetism. For the semi-classical theory to remain local, the electric charge $e$ and the magnetic charge $m$ obey the Dirac quantization condition $e m=2 \pi$, so a large $e$ (which plays the role of the coupling) can be traded for a small $m$. In general, duality maps a theory with strong coupling to one with weak coupling, and is thus a powerful tool for exploring strongly-coupled regimes.

A theory is self-dual when there is an exact map from a theory at strong coupling to the same theory at weak coupling. The best-known example occurs in the twodimensional Ising model, where the map is known as Kramers-Wannier duality, and provides a quick way to obtain the critical temperature.

In this paper, we present a exact form of self-duality in $1+1$ dimensional quantum impurity problems. This selfduality is much more powerful than in the Ising model, because there is no intermediate-coupling critical point interfering with the analytic continuation from weak to strong coupling. This duality had sometimes been used in the past as "approximate", with no clear understanding of its exact status. We show here that it results from integrability, a powerful symmetry which restricts drastically the form of the Lagrangian in the low energy limit. We make the duality very explicit by obtaining simple integral expressions for transport properties. These hint at a deep mathematical structure, somewhat similar to the one arising in supersymmetric gauge theories with Seiberg-Witten duality [1].

We study a model of $N$ flavors of interacting gapless fermions moving in one dimension. We denote the leftmoving and right-moving modes by $\psi_{L i}(x)$ and $\psi_{R i}(x)$ respectively with $i=1 \ldots N$. We consider an impurity located at $x=0$ which gives a backscattering interaction $L_{B}=\lambda \psi_{L i}^{\dagger}(0) \psi_{R i}(0)+c c$. These models can be bosonized in terms of $N$ free bosons, so that the only interaction between left- and right-moving modes is at $x=0$. For $N=1$, this is the Luttinger liquid with impurity, which in its bosonic formulation is usually called the boundary
sine-Gordon model. For $N=2$, this is a quantum wire; the two species correspond to the two spins up and down of the electrons. We consider only the case where the $N$ species are identical, and we allow for charge interactions, parametrized by a coupling constant $g(g=N$ at the free point). Since the excitations are gapless in the bulk, the only scale in the problem is the coupling $\lambda$ from the impurity interaction. There are two critical points: the "no-backscattering" point where $\lambda=0$, and the "strong-coupling" fixed point where $\lambda \rightarrow \infty$ and all the excitations are backscattered.

Let us first discuss the simplest case $N=1$, the Luttinger liquid with impurity. As is well known, the interacting fermions of the Luttinger model can be bosonized in terms of a single free boson. Moreover, one can "fold" space in half around the impurity at $x=0$ and restrict to the half-line $0<x<\infty$ [2]. Without the impurity, the Lagrangian is $L_{0}=(1 / 4 \pi g) \int_{0}^{\infty} d x\left(\partial_{\mu} \phi\right)^{2}$ where $g$ parameterizes the interactions of the original Luttinger fermions. The two critical points correspond to Neumann boundary conditions $\partial_{x} \phi(0, t)=0$ and Dirichlet boundary conditions $\phi(0, t)=$ const. As is familiar from conformal field theory, the massless boson can be decomposed into left- and right-moving pieces $\phi=\phi_{L}+\phi_{R}$. One can then define the "dual" boson $\widetilde{\phi}=\left(\phi_{L}-\phi_{R}\right) / g$ and rewrite the Lagrangian as $L_{0}=(g / 4 \pi) \int_{0}^{\infty} d x\left(\partial_{\mu} \widetilde{\phi}\right)^{2}$. Dirichlet boundary conditions on $\phi$ correspond to Neumann boundary conditions on $\widetilde{\phi}$, and vice versa. With no interactions at the boundary, the theory is therefore invariant under the mapping $g \rightarrow 1 / g$, and the interchange of Dirichlet and Neumann boundary conditions.

An interesting set of charge-carrying operators are written in terms of this boson and its dual as $\Psi_{e, m}(x, t)=$ $\exp [i e \phi(x, t)+i m \widetilde{\phi}(x, t)]$. The parameters $e$ and $m$ must be integers to ensure locality. For Euclidean time this entire problem can be reformulated as a classical two-dimensional Coulomb gas [3], where $\Psi_{e, 0}$ creates an electrical charge $e$, and $\Psi_{0, m}$ creates a magnetic charge $m$. In this context, the condition that $e$ and $m$ be integers is analogous to the Dirac quantization condition. The boundary two-point function is 
$\left\langle\Psi_{e, m}(0,0) \Psi_{-e,-m}(0, t)\right\rangle \sim t^{-2 x_{e, m}}$ where the boundary scaling dimension $x_{e, m}=(e \sqrt{g}+m / \sqrt{g})^{2}$, which has the electric-magnetic duality $g \rightarrow 1 / g$.

Including the impurity interaction causes operators to be added to the Lagrangian. A relevant boundary operator has boundary dimension $x_{e, m}<1$. Without loss of generality we can consider $g<1$, because any $g>1$ model can be mapped to a $g<1$ model by sending $g \rightarrow 1 / g$ and exchanging the two fixed points. This duality is not self-duality; it does not say anything about the $g<1$ models by themselves but only that the $g>1$ models are equivalent. When $g<1$, only operators with $m=0$ are relevant [ $\mid \mathbb{1}$. When $g \geq 1 / 4$, only $\Psi_{1,0}$ and its conjugate $\Psi_{-1,0}$ are relevant. Therefore, we consider the backscattering Lagrangian

$$
L_{B}=\lambda\left(\Psi_{1,0}+\Psi_{-1,0}\right)=2 \lambda \cos \phi(0, t) .
$$

This interaction induces a flow from Neumann boundary conditions (UV) to Dirichlet (IR) as $\lambda$ increases, or, equivalently, as energy decreases. From this expression one finds for weak backscattering at zero temperature, the current $I$ and voltage $V$ obey the scaling law $I-g V \propto V^{2 g-1}$ 四.

The self-duality appears in the action in the strong coupling limit. At very large $\lambda$, the field $\phi(0, t)$ is pinned at the minima of the potential, so it is reasonable to study the vicinity of the Dirichlet fixed point by considering the instantons interpolating between neighboring minima [5]. After some elementary computations, one obtains the leading (charged) irrelevant operator

$$
L_{B} \approx \lambda_{D}\left(\Psi_{0,1}+\Psi_{0,-1}\right)=2 \lambda_{D} \cos \widetilde{\phi}(0, t) .
$$

The subscript $D$ is for dual, because this form of the backscattering lagrangian is dual to the form (11). Since $\cos \widetilde{\phi}(0, t)$ has dimension $1 / g, \lambda_{D} \propto \lambda^{-1 / g}$, and at zero temperature the current and voltage obey the scaling law $I \propto V^{2 / g-1}$ in the strong-backscattering limit [4]. Thus the strong and weak-backscattering exponents are related by interchanging $g \rightarrow 1 / g$.

If (2) were exact, the theory would be completely selfdual under the interchange $\lambda \rightarrow \lambda_{D}$ and $g \rightarrow 1 / g$. This has been often assumed in the literature, and is of course very appealing, especially in the context of the fractional quantum Hall effect at $\nu=1 / 3$. Here, the Luttinger fermions at $g=1 / 3$ are equivalent to Laughlin quasiparticles, and the impurity corresponds to a point contact allowing tunneling between edges of a fractional quantum Hall device [6]. In the weak-backscattering limit, the tunneling (11) is caused by Laughlin quasiparticles. In the strong backscattering limit the system is effectively two different Hall devices, weakly coupled by (2) so that only electrons can tunnel. Thus the duality maps a description in terms of Laughlin quasiparticles to one in terms of electrons.
However, there is no reason a priori why the duality should be exact: the instanton expansion of [5] is not exact, and multi-instanton processes can be expected to contribute, adding to (2) terms $\propto \cos n \widetilde{\phi}(0, t)$. In addition, neutral operators will also contribute, for instance the density - density coupling $(\partial \widetilde{\phi})^{2}$ (which has dimension 2 and so for $g<1 / 2$ is the leading irrelevant operator). The perturbative computations near the strong coupling fixed point will involve all of these terms, and thus have little to do with its weak-coupling dual (1).

It turns out however that Bethe ansatz computations 22 established that there is indeed an exact duality for the current at zero temperature:

$$
I(\lambda, g, V)=g V-g I\left(\lambda_{D}, \frac{1}{g}, g V\right)
$$

Some well-motivated and well-checked conjectures require that this also hold at arbitrary $T$ [7]. The selfduality relation (3) can be derived directly by imposing the strong constraints of integrability on the IR action. The integrability of the boundary sine-Gordon model means that there are an infinite set of conserved quantities in involution, even with the perturbation (11) of the UV fixed point. These are generated by the non-local operator $\cos \widetilde{\phi}$, and by an infinite set of local operators $\mathrm{O}_{2 k+2}$ which are, roughly, powers of the stress energy tensor, with dimensions $2 k+2, k$ an integer. By considering the Yang-Baxter equation and its various algebraic consequences in detail [7] 10], or more simply by demanding integrability of the flow both near the UV and near the IR fixed point, it can be shown that the complete IR lagrangian is given by (2) plus an infinite series of the type $\sum_{k=0}^{\infty} c_{k} \lambda_{D}^{(2 k+1) g /(1-g)} \mathrm{O}_{2 k+2}$ (this sort of statement of course can only make sense within a particular regularization scheme, here dimensional regularization [10]). The remarkable fact is that only one charged operator appears. It is also crucial that the neutral operators all commute: as a result, it can be shown, by using the non equilibrium Keldysh formalism, that DC transport properties are insensitive to anything but (2), and thus are self-dual, at any temperature. Therefore (3) must hold to all orders in perturbation theory.

The existence of the duality prompts questions about the analytic structure of the whole problem: knowing a priori that (3) holds, could one find the exact formula for $I$ without using the Bethe ansatz? We present here a formulation of the problem that suggests it should be the case, and bears an intriguing resemblance with |1] (for a previous attempt in a different direction, see [8]). Introducing the dimensionless parameter $u \propto V \lambda^{1 /(g-1)}$ (the precise relation is given in [2]), doing perturbation theory in powers of $\lambda$ in (11) gives

$$
\frac{I}{g V}=1-\sum_{n=1}^{\infty} a_{n}(g) u^{2 n(g-1)}
$$


for large $u$ (small $\lambda)$. The analogous expansion in the strong-backscattering regime (small $u$ ) must be

$$
\frac{I}{g V}=\sum_{n=1}^{\infty} a_{n}(1 / g) u^{2 n(1 / g-1)}
$$

because the self-duality relation (3) means that not only the powers but also the coefficients in the expansions of the current are dual under $g \rightarrow 1 / g$. Considering the dimensionless current $\mathcal{I}(g, u) \equiv I / g V$ as an analytic function of $g$ gives the non-perturbative self-duality

$$
\mathcal{I}(g, u)=1-\mathcal{I}(1 / g, u) .
$$

This equation applies for all $g$, so does much more than just relate a model at $g>1$ to one with $g<1$.

Given either one of the expansions (4) or (5), we can now write an expression for the current which not only makes proving the self-duality simple, but which displays an intriguing connection to Seiberg-Witten theory. The Bethe ansatz gives the coefficients $a_{n}$ to be [2]

$$
a_{n}(g)=\frac{(-1)^{n+1}}{n !} \frac{\sqrt{\pi}}{2} \frac{\Gamma(n g+1)}{\Gamma(3 / 2+n(g-1))}
$$

With this $a_{n}$, the expansions (画) and (5) have finite radius of convergence, so the self-duality must hold nonperturbatively. Simple expressions for the magnetization can be found by using the integral form of the $B$ function $\Gamma(a) \Gamma(b) / \Gamma(a+b)$ to rewrite $a_{n}(g)$. Since the sum (㺼) is absolutely convergent for large enough $u$, we can interchange the order of summation and integration, and do the sum. This yields our result

$$
\mathcal{I}(g, u)=\frac{i}{4 u} \int_{\mathcal{C}_{0}} d x \frac{1}{\sqrt{x+x^{g}-u^{2}}}
$$

where the contour of integration $\mathcal{C}_{0}$ starts at the origin, loops around the square-root branch point on the positive real axis, and returns to the origin. There must be such a branch point because the expression inside the square root is positive for large $x$ and negative at $x=0$. By using the fluctuation-dissipation theorem $\left\langle(\Delta I)^{2}\right\rangle=\left((g V)^{2} /(1-g)\right) \partial_{V} \mathcal{I}$ derived in [9], one obtains an integral expression for the DC shot noise as well.

It can easily be checked that the series (4) can be recovered from (8) by changing variables in the integral to $t=x / u^{2}$, and expanding the resulting integrand in powers of $u^{2 g-2}$. However, the integral in (8) converges for all real $u$, not just in the large- $u$ regime where the sum (伍) does. Therefore it must also yield the expansion (5) in the small- $u$ limit. By defining the variable $t=x / u^{2 / g}$ and expanding the resulting integrand in powers of $u^{2 / g-2}$, one can indeed verify this fact. Moreover, one can prove the self-duality relation (6) without the series expansions, because the integral expression (8) is analytic in $g$ and so can be utilized for any $g$ :

$$
\begin{aligned}
\mathcal{I}(1 / g, u) & =\frac{i}{4 u} \int_{\mathcal{C}_{0}} d x \frac{1}{\sqrt{x+x^{1 / g}-u^{2}}} \\
& =\frac{i}{4 u} \int_{\mathcal{C}_{0}} d x \frac{g x^{g-1}}{\sqrt{x+x^{g}-u^{2}}} \\
& =\frac{i}{2 u} \int_{\mathcal{C}_{0}} d x \frac{\partial}{\partial x}\left[\sqrt{x+x^{g}-u^{2}}\right]-\mathcal{I}(g, u) \\
& =1-\mathcal{I}(g, u)
\end{aligned}
$$

where to get from the first to second line we change variables $x \rightarrow x^{g}$, and $\mathcal{C}_{0}$ changes accordingly.

Our expressions for the current bear a strong resemblance with the representations obtained in [1] for quantities such as mass gap in $N=2$ supersymmetric gauge theory. There the parameter $u$ is an order parameter related to the expectation value of the Higgs field. This is perhaps not so shocking. The idea of Seiberg-Witten theory is to exploit the fact that certain physical quantities are analytic in some coupling, except at specific singularities. If one takes the coupling around a singularity, physical quantities do not necessarily return to their original values (for example if they have logarithmic behavior near the singularity): there is a non trivial Berry's phase, or monodromy. If one knows all the singularities and monodromies of an otherwise-analytic function, one can reconstruct the complete function for any value of the coupling: its typical form in the present context is given by $\int d x / y$, where $y^{2}$ is a rational function of $x$, and the integral loops around some of the square-root branch points of the integrand. The coefficients in $y^{2}$ depend on the couplings of the theory. Singularities occur at values of the couplings where two roots of $y^{2}$ coincide, so that the integral logarithmically diverges. In the gauge theories discussed in [1] and in many subsequent generalizations, one can use perturbation theory to find the monodromies. Thus perturbative information and analyticity leads to non-perturbative results.

In integrable models, analyticity in couplings has long been exploited to do various computations [11]. In the present context, the analyticity is in the dependence of the current on complex $u$ (that is, voltage). Little seems to be known in general about this question, but the integral expression (8) reveals a very simple structure. The current is singular at the values $u_{0}$ where both $x+x^{g}-u_{0}^{2}=0$ and $1+g x^{g-1}=0$, namely $u_{0}^{2}=(-g)^{g /(1-g)}(1-g)$. When $u=u_{0}$, two of the roots of $x+x^{g}-u^{2}$ coalesce. The integral (8) diverges if the contour runs in between the two coalescing roots; this never happens for positive real $u$. Thus we know the exact location of all of the Lee-Yang zeroes of the current. All of them have the same absolute value $\left|u_{0}\right|$, and for rational values of $g$, there are a finite number (for some reasons why this is, see [12]). The value $\left|u_{0}\right|$ gives the radii of convergence: the series (4) absolutely converges for $|u|>\left|u_{0}\right|$, while (5) absolutely converges for $|u|<\left|u_{0}\right|$. 
There are also non-trivial monodromies in this problem that can be extracted from the integral expressions. For example, when $g=1 / 2$, the integral can be done to find the known result $\mathcal{I}=1 / 2-(1 / 2 u) \arctan (2 u)$. This has singularities at $u_{0}= \pm i / 2$. Looping $u$ around one of the singularities moves onto a different sheet of the arctangent, so $\mathcal{I} \rightarrow \mathcal{I} \pm \pi /(2 u)$ for $g=1 / 2$. Unfortunately, for general $g$ the monodromies are more complicated and at the present time we do not know how to obtain them without already knowing the integral (8).

The foregoing self-duality extends to the case of $N$ channels. The case $N=2$, a quantum wire with impurity, was studied perturbatively in [4], and the exact current was found using the Bethe ansatz in [13. Following 2,13, it is straightforward to find the large- $u$ series expansion of the current for general $N$. Defining as before $\mathcal{I}_{N}=I / g V, g$ the conductance without impurity $(g=N$ for $N$ channels of free electrons), one finds

$$
\mathcal{I}_{N}=\sum_{n=0}^{\infty}(-1)^{n} \frac{\sqrt{\pi}}{2} \frac{\Gamma(n(1+N h)+1) \Gamma(n h+1)}{n ! \Gamma(n N h+1) \Gamma(n h+3 / 2)} u^{2 n h}
$$

where $h=\frac{1}{g}-\frac{1}{N}$. This can be written as the integral

$$
\mathcal{I}_{N}(g, u)=\frac{i}{4 u} \int_{\mathcal{C}_{0}} d x \frac{\left(1+x^{h}\right)^{N-1}}{\sqrt{x\left(1+x^{h}\right)^{N}-u^{2}}}
$$

This normalized current obeys the self-duality relation (6) as with the $N=1$ case, here with the dual coupling $N^{2} / g$. It would be quite interesting to see whether this result generalizes to the models discussed in [14].

Duality considerations extend beyond transport properties; for instance, it is known from the exact solution that there was a sort of duality between the UV and IR in the Kondo model [15]. This follows the same pattern as before. Consider the anisotropic Kondo problem, which is defined by the boundary Lagrangian generalizing (1), $L_{B}=\lambda\left(\Psi_{1,0} S_{j}^{-}+\Psi_{-1,0} S_{j}^{+}\right)$, where $S^{ \pm}$are $s u(2)_{q}$ spins $\left(q=e^{i \pi g}, g\right.$ parameterizing the anisotropy) in the representation of spin $j$. As with the tunneling problem, the integrability requires the IR Lagrangian to be very simple: it contains only the conserved quantities $O_{2 k+2}$ and a single non-trivial term like (2): $L_{B} \approx$ $\lambda_{D}\left(\Psi_{0,1} S_{j-1 / 2}^{-}+\Psi_{0,-1} S_{j-1 / 2}^{+}\right)$, where now the spins are in the representation of spin $j-1 / 2$. As a result, the free energy at magnetic field $H$ satisfies a duality relation similar to (6)

$$
f(j, \lambda, g, H)=f\left(j-\frac{1}{2}, \lambda_{D}, \frac{1}{g}, \frac{H}{g}\right)+\ldots
$$

The left-hand side is the expansion in the UV, whereas the right-hand side is the IR expansion; the extra terms on the right-hand side are only in odd-integer powers of $H / \lambda^{1 / g-1}$, and arise because the free energy does couple to the charge-neutral operators in the IR action. The free energies for all $S$ are also given by integrals like (8), which surround branch points in the complex plane [12].

There are some interesting distinctions between these quantum-impurity problems and the Seiberg-Witten theories. In the gauge theories, couplings never seem to appear in the exponents of $x$. Here $g$ rational leads to a polynomial for $y^{2}$ as in the gauge theories, but $g$ irrational is a slightly new situation. However, this is quite interesting because none of the $g \rightarrow 1 / g$ dualities known in gauge theory have a proof like that in (9). Moreover, the Luttinger liquid with impurity is not supersymmetric; as argued above the analyticity seems to follow from the integrability, not from supersymmetry as in the gauge theories. This opens up a new realm of possibilities in gauge theory, a few of which are discussed in 12 .

In conclusion, it is likely that duality is a key feature of integrable field theories in $1+1$ dimensions. For example, a situation similar to what we have discussed should occur in the massless flows between minimal models of conformal field theory, where we expect a duality between the $\phi_{13}$ perturbations in the ultraviolet and $\phi_{31}$ perturbations in the infrared.

[1] N. Seiberg and E. Witten, Nucl. Phys. B426 (1994) 19, hep-th/9407087; Nucl. Phys. B431 (1994) 484, hepth/9408099

[2] P. Fendley, A.W.W. Ludwig and H. Saleur, Phys. Rev. B52 (1995) 8934, cond-mat/9503172

[3] For a review see B. Nienhuis in Phase Transitions and Critical Phenomena vol. 11, ed. C. Domb and J. Lebowitz

[4] C.L. Kane, M.P.A. Fisher, Phys. Rev. B46 (1992) 15233

[5] A. Schmid, Phys. Rev. Lett. 51 (1983) 1506

[6] for a review, see C.L. Kane and M.P.A. Fisher in Perspectives in Quantum Hall Effects, ed. by S. Das Sarma and A. Pinczuk (Wiley, 1997)

[7] V. Bazhanov, S. Lukyanov and A.B. Zamolodchikov, Comm. Math. Phys. 190 (1997) 247, hep-th/9604044

[8] U. Weiss, Solid State Comm. 100 (1996) 281, condmat/9604027

[9] P. Fendley, A.W.W. Ludwig and H. Saleur, Phys. Rev. Lett. 75 (1995) 2196, cond-mat/9505031

[10] F. Lesage, H. Saleur, "Duality and IR perturbation theory in quantum impurity problems", to appear

[11] R.J. Baxter, Exactly solved models in statistical mechanics, (Academic Press, 1982)

[12] P. Fendley, "Duality Without Supersymmetry", hepth/9804108

[13] F. Lesage, H. Saleur and P. Simonetti, cond-mat/9703220

[14] H. Yi and C.L. Kane, cond-mat/9602099; C. Nayak, M.P.A. Fisher, A.W.W. Ludwig and H.-H. Lin, condmat/9710305

[15] V. Fateev and P. Wiegmann, Phys. Lett. 81A (1981) 179 